\title{
A bulged lin-4/lin-14 RNA duplex is sufficient for Caenorhabditis elegans lin-14 temporal gradient formation
}

\author{
Ilho Ha, Bruce Wightman, ${ }^{1}$ and Gary Ruvkun ${ }^{2}$ \\ Department of Molecular Biology, Massachusetts General Hospital, and Department of Genetics, Harvard Medical School, \\ Boston, Massachusetts 02114 USA
}

The Caenorhabditis elegans heterochronic gene lin-14 generates a temporal gradient of the LIN-14 proteins to control stage-specific patterns of cell lineage during development. Down-regulation of LIN-14 is mediated by the lin-14 $3^{\prime}$ untranslated region (UTR), which bears seven sites that are complementary to the regulatory lin-4 RNA. Here we report molecular and genetic evidence that RNA duplexes between the lin-4 and lin-14 RNAs form in vivo and are necessary for LIN-14 temporal gradient generation. lin-4 RNA binds in vitro to a lin-14 mRNA bearing the seven lin-4 complementary sites but not to a lin-14 mRNA bearing point mutations in these sites. In vivo, the lin-4 complementary regions are necessary for lin-14 3' UTR-mediated temporal gradient formation. Based on lin-14 3' UTR sequence comparisons between C. elegans and C. briggsae, four of the seven lin-4/lin-14 RNA duplexes are predicted to bulge a lin-4 C residue, and three sites are predicted to form nonbulged RNA duplexes. Reporter genes bearing multimerized bulged $\mathrm{C}$ lin- 4 binding sites show almost wild-type temporal gradient formation, whereas those bearing multimerized nonbulged lin- 4 binding sites do not form a temporal gradient. Paradoxically, lin-4 RNA binds in vitro to nonbulged lin-14 RNA more avidly than to the bulged lin-14 RNA. This suggests that a specific secondary structure of lin-4/lin-14 RNA duplex that may be recognized by an accessory protein, rather than an RNA duplex per se, is required in vivo for the generation of the LIN-14 temporal gradient.

[Key Words: C. elegans; development; heterochronic; bulged RNA; translation; lin-14, lin-4; 3' UTR]

Received August 26, 1996; revised version accepted October 21, 1996.

Genetic and molecular analyses in Drosophila and Caenorhabditis elegans have shown that gene pathways specific to one axis of the developing animal-for example, anterior/posterior (Nüsslein-Volhard 1991), dorsal/ventral (Rushlow et al. 1988; Serano and Cohen 1995), or temporal (Ruvkun and Giusto 1989)—generate control gene activities that form a gradient over that developmental axis to specify patterned arrays of cells. In many cases, these pattern formation genes have been shown to be regulated post-transcriptionally (Wang and Lehmann 1991; Wharton and Struhl 1991). The C. elegans heterochronic genes regulate the temporal sequence of the cell lineage by such a mechanism. These genes post-transcriptionally generate a temporal gradient of a key regulator, the LIN-14 protein (LIN-14) (Ambros 1989; Ruvkun and Giusto 1989; Wightman et al. 1993).

During wild-type $C$. elegans development, blast cells execute stage-specific patterns of cell lineage at each of the four larval stages and terminally differentiate during the adult stage (Ambros and Horvitz 1984). Mutations in

1Present address: Biology Department, Muhlenberg College, Allentown, Pennsylvania 18104 USA.

${ }^{2}$ Corresponding author. the heterochronic genes lin-4, lin-14, lin-28, and lin-29 cause temporal transformations in these stage-specific patterns of cell lineage in many tissues and cell types (Chalfie et al. 1981; Ambros and Horvitz 1984). Depending on the heterochronic mutant, stage-specific developmental events occur at either earlier or later stages than they would normally.

An instructive role for lin-14 in temporal pattern formation was established by the observation that lin-14 gain-of-function and loss-of-function mutations have the opposite phenotype (Ambros and Horvitz 1984). Loss-offunction (lf) lin-14 alleles cause precocious execution at early larval stages of cell fates appropriate for later larval stages (Ambros and Horvitz 1984). Gain-of-function (gf) lin-14 alleles cause the opposite transformation in temporal cell fate-reiterations of early cell fates at later stages.

lin-14 controls stage-specific cell lineages by generating a temporal gradient of the LIN-14 nuclear proteins (Ruvkun and Giusto 1989). The high level of LIN-14 at early stages specifies an early cell lineage, and the falling level of the LIN-14 causes a switch to late cell lineages. The normal temporal regulation of LIN-14 abundance is required for the wild-type sequence of temporal cell 
fates. Heterochronic mutations that elevate LIN-14 levels at late stages cause reiterations of early cell lineages, whereas mutations that decrease LIN-14 levels at early stages cause precocious expression of late cell lineages (Ruvkun and Giusto 1989; Arasu et al. 1991; Wightman et al. 1991).

Temporal regulation of LIN-14 abundance during wild-type development occurs at a post-transcriptional step (Wightman et al. 1993). This post-transcriptional regulation is mediated by the lin-14 $3^{\prime}$ untranslated region (UTR) that is sufficient to confer such regulation on an unrelated reporter gene (Wightman et al. 1993). lin-4 gene activity is required for the post-transcriptional temporal regulation of $\operatorname{lin}-14$ or reporter genes bearing the lin-14 3' UTR. In the region of the lin-14 3' UTR that is deleted in $l$ in -14 (gf) mutations are seven copies of a 14to 19-nucleotide sequence that is complementary to a portion of the regulatory lin-4 RNA (Lee et al. 1993). This suggests that lin- 4 down-regulates lin-14 translation by forming multiple RNA duplexes with the lin-14 mRNA (Wightman et al. 1993).

Here we show that multiple elements in the lin-14 3' UTR are conserved between C. briggsae and C. elegans, but that the lin- 4 complementary elements account for the lin-14 temporal gradient forming activity. A lin-4/ lin-14 RNA duplex forms in vitro. In vivo, particular lin-4/lin-14 RNA duplexes that bulge a nucleotide are active in temporal gradient formation, whereas nonbulged RNA duplexes are not. We propose that a specific RNA secondary structure is required for post-transcriptional generation of the LIN-14 temporal gradient.

\section{Results \\ Conserved elements in the lin-14 3' UTR}

Comparison of nucleic acid sequences between C. elegans and the related nematode $C$. briggsae species has successfully identified regulatory elements by their DNA sequence conservation (Zucker-Aprison and Blumenthal 1989; Heschel and Baillie 1990; Xue et al. 1992; Lee et al. 1993). The C. briggsae lin-14 3' UTR mediates formation of the LIN-14 temporal gradient in C. briggsae, and a lacZ reporter gene fused to the C. elegans lin-14 3' UTR forms a temporal gradient in C. briggsae (Wightman et al. 1993). Therefore, those sequences that are required for lin-14 temporal regulation should be conserved between the two species. Comparison of the C. briggsae and C. elegans lin-14 3' UTR sequences reveals multiple blocks of conserved sequence, separated by stretches with no conservation (Fig. 1A). The longest stretch of exactly conserved sequence is 30 nucleotides (24250-24280). The region that is deleted by both lin14(gf) mutations (24168-25516) contains conserved blocks of various lengths scattered throughout. Additional conserved blocks of sequence are found between the deletion breakpoint of the weaker gf mutation (n536) and the polyadenylation signal (24819-25515). The position and stem complementarity of the most stable stem- loop structure is also conserved (Fig. 1A). In contrast, almost no sequence conservation was found in the 600 bases immediately $3^{\prime}$ to the $C$. elegans stop codon (position 23912), in the region $3^{\prime}$ to the polyadenylation signal, and in introns, suggesting that these regions may be dispensable for lin-14 function.

Seven repeated 9- to 15-nucleotide sequences that are complementary to a portion of the lin-4 RNAs are completely conserved between the two species (Fig. 1B). Because the portion of the lin-4 RNAs that is complementary to these repeated elements is also completely conserved between $C$. elegans and C. briggsae (Lee et al. 1993), this result is consistent with the model that multiple lin-4 RNAs hybridize to the 3' UTR of the lin-14 mRNA.

\section{A lin-4/lin-14 RNA duplex forms in vitro}

To observe RNA duplex formation in vitro, a ${ }^{32} \mathrm{P}$-labeled chemically synthesized 24-nucleotide lin-4 RNA that corresponds to the major lin-4 transcript (Lee et al. 1993) was incubated with in vitro transcribed lin-14 3' UTR RNA (nucleotides 23845-25637) bearing the seven putative lin- 4 binding sites, and complexes were resolved by native gel electrophoresis (Fig. 2). The wild type lin-4 RNA binds to wild-type lin-14 3' UTR RNA but not to a lin-14 (7Xmut) 3' UTR RNA that bears helix-disrupting point mutations (UCA to AGU; Fig. 1B) in each of the seven lin-4 complementary regions (Fig. 2). Mutant lin4(ma161) RNA carrying the C to U lin-4(ma161) base substitution in the proposed lin-14 complementary region (at lin-4 nucleotide number 5 in Fig. 1B) shows lower affinity than the lin-4(+) RNA to the wild-type lin-14 3' UTR RNA, and no binding to the lin-14(7Xmut) 3' UTR RNA (Fig. 2). This in vitro data shows that the postulated RNA duplex between the lin-4 and lin-14 RNAs in fact forms. In addition, it suggests that the strong lin-4(ma161) allele affects the stability of the lin4/lin-14 RNA duplex, consistent with thermodynamic calculations of the effects of the $\mathrm{C}$ to $\mathrm{U}$ transition on duplex formation (Lee et al. 1993; Wightman et al. 1993). Because these same mutations in the lin-14 $3^{\prime}$ UTR affect its function in vivo (see below), this in vitro assay may reflect the in vivo function of these RNAs.

\section{Mutations that disrupt in vitro formation of} lin-4/lin-14 RNA duplex disrupt temporal gradient formation in vivo

To further test the lin-4/lin-14 RNA duplex model, we compared in vivo temporal gradient formation by the lin-14(7Xmut) 3' UTR, bearing helix-disrupting mutations in each of the seven proposed lin- 4 binding sites, to that of the wild-type lin-14 $3^{\prime}$ UTR. Temporal gradient formation was compared in animals expressing wildtype lin-4 RNA as well as in the lin-4(e912) strain that deletes the entire lin-4 region (Lee et al. 1993). To suppress any indirect effects on temporal gradient formation resulting from the heterochronic effects of the lin- $4 \mathrm{mu}$ tant from direct effects on the lin-14 3' UTR, hetero- 
A

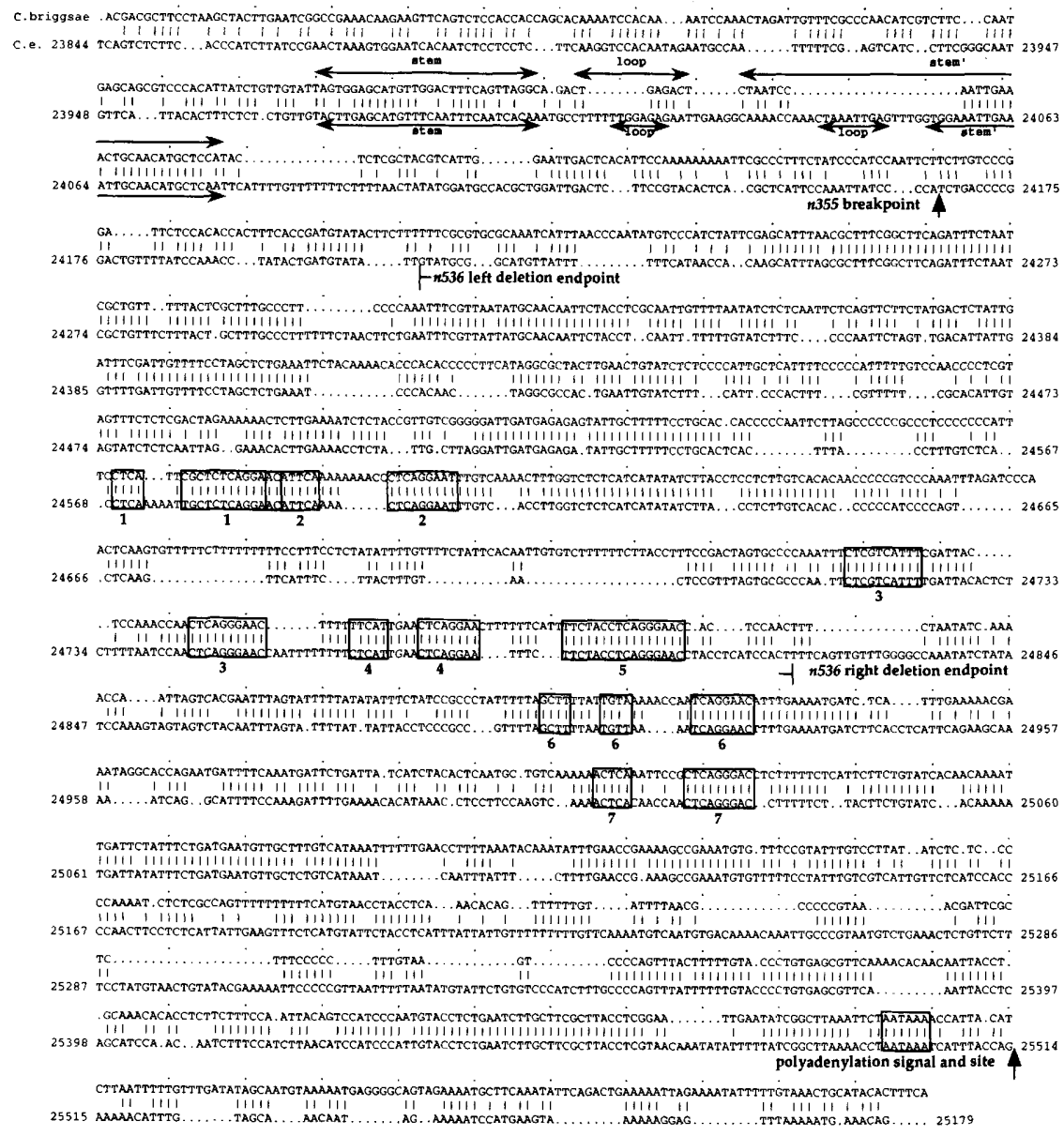

B

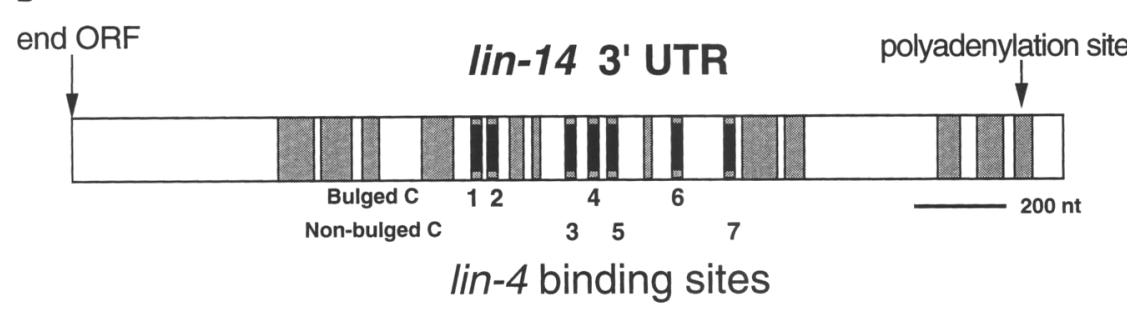

Bulged Duplex

Nonbulged Duplex

2

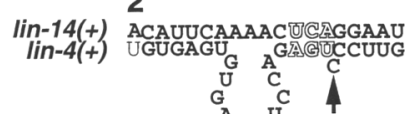

2

lin-14(7Xmut.) ACAUUCAAAACAGEGGAAU lin-4(t) UGUGAGUG<smiles>CC(C)=CCOCCOCCOCCO</smiles>

2

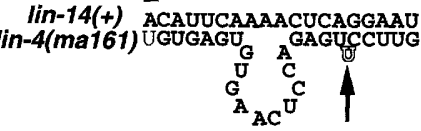

Figure 1. (A) A comparison of the lin-14 3' UTR sequences from $C$. elegans and $C$. briggsae. The top line shows the $C$. briggsae sequence /GenBank accession no. U67400); the bottom line shows the C. elegans sequence (Wightman et al. 1991). Sequences that are conserved are shown with dashed lines between the C. briggsae and C. elegans sequences. Potential lin- 4 binding sites are designated by boxes that are numbered to correspond to those shown in detail in Fig. 1B. The lin-14(gf) mutations and polyadenylation site for the C. elegans sequence are labeled below the line. Positions in this figure are numbered according to genomic sequences of cosmid $\mathrm{T} 25 \mathrm{C} 12$, which contains the lin-14 gene. Dots indicate gaps introduced into both sequences to allow alignment. The most stable stem and loop structure are shown as doubleheaded arrows (stem and stem', loop). In both C. elegans and C. briggsae, this stemloop has the lowest predicted free energy of any predicted stem-loop in the $3^{\prime}$ UTR $(-38.3$ and $-27.3 \mathrm{kcal} / \mathrm{mole}$, respectively) (Jaeger et al. 1989). In the region of the stems the sequence is conserved between the two species. At most positions where the sequence differs between the two species, compensatory changes in the other half of the stem would maintain base-pairing. This observation suggests that a stemloop structure may be required at this position, but that a particular sequence may not be required. This $C$. elegans stem-loop structure is not deleted in either lin-14(gf) mutation, indicating that the structure is not sufficient for temporal regulation, and the analysis below suggests that it is not necessary for temporal gradient formation. (B) Locations of the proposed lin-14 3' UTR RNA/lin-4 RNA duplexes and other conserved regions in the lin-14 3' UTR. Four out of seven lin-4 binding sites $(1,2,4$, and 6) bulge a lin-4 $\mathrm{C}$ from the duplex (arrow). Examples of a bulged C lin-4/lin-14 RNA duplex (\#2) and a nonbulged RNA duplex $(\# 5)$ and the alterations of these structures predicted from lin-14(7Xmut) 3' UTR (underlined outline AGU) and the lin4(ma161) RNA sequence (outline $U$ ) are shown. 
Ha et al.

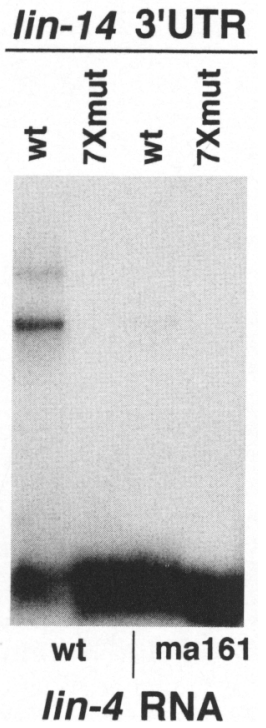

Figure 2. In vitro binding analysis of lin-4 RNA and lin-14 3' UTR RNA. lin-14 RNAs were prepared by in vitro transcription from a T3 promoter, and lin-4 RNAs were synthesized chemically. ${ }^{32}$ P-labeled lin-4 RNA was incubated with lin-14 3' UTR RNA at room temperature and analyzed by agarose gel $(1 \%)$ electrophoresis. Unbound labeled lin-4 RNA migrates to the bottom of the gel, whereas lin-4/lin-14 RNA complexes migrate more slowly. The lin-4(+) RNA binds to a lin-14(+) RNA but not to the lin-14(7Xmut) RNA carrying mutations in each of the seven lin-4 binding sites. lin-4(ma161) RNA binds lin-14(+) RNA more weakly. The amount of lin-14 RNAs for each lane is equivalent as measured by binding to a DNA oligonucleotide outside of the lin-4 complementary region (data not shown).

chronic defects were suppressed by a lin-14(n179ts) point mutation in the protein coding region (B. Reinhart and G. Ruvkun, pers. comm.), which is benign in this assay (Fig. 3). Whereas transgenic animals carrying a $l a c Z$ reporter gene fused to a wild-type lin-14 $3^{\prime}$ UTR show lin-4-dependent down-regulation from the $\mathrm{L} 1$ to $\mathrm{L} 4$ stage (130-fold), animals bearing the reporter gene fused to the lin-14(7Xmut) 3' UTR bearing helix-breaking mutations in each of the seven lin-4 complementary regions show only 8 -fold down-regulation that is not lin-4 dependent (Fig. 3). The 8-fold down-regulation of the lin14(7Xmut) 3' UTR is equivalent to the level of lin-4independent down-regulation shown by the control unc$543^{\prime}$ UTR (Wightman et al. 1993).

Thus a lin-14(7Xmut) 3' UTR that is specifically disrupted in the seven lin-4 complementary sites is 16-fold less active in temporal gradient formation than the wildtype lin-14 3' UTR (Fig. 3). Similarly, temporal gradientforming activity of the wild-type lin-14 3' UTR in a lin-4 mutant background is decreased 5- to 30 -fold relative to wild type, whereas lack or presence of lin-4 gene activity has no effect on the lin-14 3' UTR lacking lin-4 binding sites (Fig. 3). Thus all of the lin-14 3' UTR temporal gradient forming activity detectable by this fusion gene assay is mediated by the lin-4 complementary region of the lin-14 3' UTR. This data supports the model that the

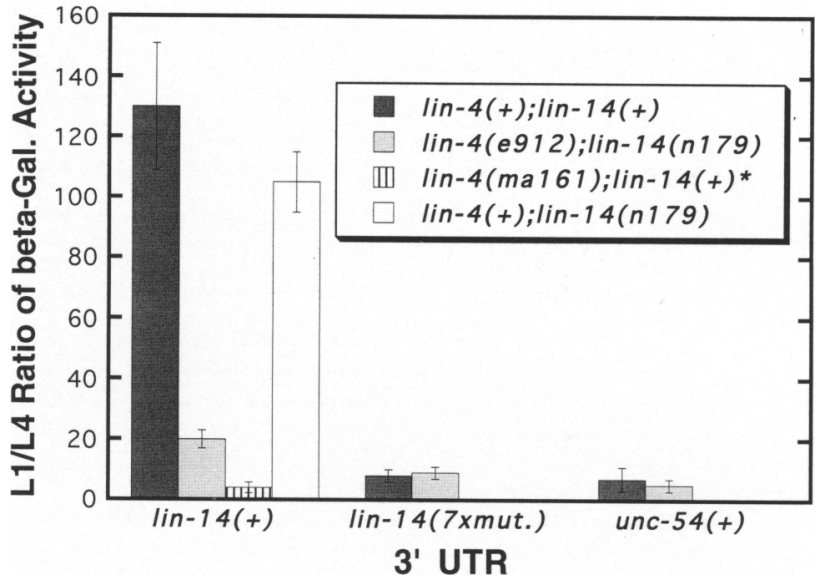

Figure 3. Down-regulation of $\beta$-galactosidase activity in transgenic wild-type or lin-4 mutant animals carrying reporter genes fused to the lin-14(+), lin-14(7Xmut), or control unc-54 3' UTRs. The $y$-axis represents the ratio of $\beta$-galactosidase activity in L1 stage animals relative to L4 stage animals. The same transgenic arrays that are analyzed in wild type were transferred by mating to the lin- 4 mutant background, so that differences between temporal gradient formation in lin-4(+) and lin-4 mutant backgrounds are not a result of differences in the transgenic arrays. $\left({ }^{\star}\right)$ The integrated line (pC10UT; Wightman et al. 1993) was analyzed in the lin-4(ma161) genetic background. To uncouple reporter gene activities from indirect effects of $l i n-4$ on temporal cell fates mediated by heterochronic lin-14 activity, fusion gene expression was monitored in a lin-4(e912);lin$14(\mathrm{n} 179 \mathrm{ts})$ strain at $20^{\circ} \mathrm{C}$. At this temperature, the temperaturesensitive LIN-14(n179ts) protein suppresses the lack of lin-4 mediated LIN-14 down-regulation and vice versa, sô that animals show no heterochronic Lin-4 or Lin-14 phenotype. Temporal regulation of reporter gene expression in lin-4(e912); lin$14(n 179$ ts $)$ at $20^{\circ} \mathrm{C}$ was very similar to that observed in lin4(e912) background (Wightman et al. 1993). The disruption of down-regulation in lin-4(e912); lin-14(n179ts) is not a result of the lin-14(n179ts) mutation; the same lin-14(+) 3' UTR reporter gene in lin-14(n179ts) shows 105-fold temporal downregulation, which is similar to down-regulation of this transgene in wild type. Animals carrying the lin-14(7Xmut) 3' UTR reporter gene show negligible temporal gradient generation activity in either lin-4(e912); lin-14(n179ts) (9-fold) or wild type (8-fold). The residual 3- to 8-fold down-regulation of reporter genes bearing the unc-54 3' UTR or the lin-14(7xmut) $3^{\prime}$ UTR may reflect the temporal regulation of the collagen promoter or the surface area-to-volume ratio dilution effect of the larger L4 animals. The specific $\beta$-galactosidase activities $[\Delta$ optical density (O.D.) $574 / \mathrm{min} . / \mathrm{mg}$ protein] of the transgene with wildtype $\operatorname{lin}-143^{\prime}$ UTR are $2.61 \pm 0.30(\mathrm{~L} 1)$ and $0.018 \pm 0.002$ (L4) $\left[\operatorname{lin}-4(+)_{j} \operatorname{lin}-14(+)\right], 11.18 \pm 0.84$ and $0.57 \pm 0.074$ [lin-4(e912); lin-14(n179ts)], $0.99+ \pm 0.37$ and $0.23 \pm 0.02$ [lin-4(ma161); lin$14(+), 6.85 \pm 0.15$ and $0.066 \pm 0.006$ [lin-4 $\left.(+)_{;} \operatorname{lin}-14(n 179 t s)\right]$. For transgenes of lin-14(7Xmut $) 3^{\prime}$ UTR they are $2.0 \pm 0.67$ and $0.24 \pm 0.055\left[\operatorname{lin}-4(+)_{i} \operatorname{lin}-14(+)\right]$ and $4.5 \pm 0.93$ and $0.48 \pm 0.058$ [lin-4(e912); lin-14(n179ts)]. For transgenes with unc-54 3' UTR they are $9.0 \pm 4.4\left[\operatorname{lin}-4(+)_{i} \operatorname{lin}-14(+)\right]$ and $1.4 \pm 0.7$ [lin4(e912); lin-14(n179ts)] (Wightman et al. 1993).

duplex formation between lin-4 RNA and lin-14 3' UTR is essential for formation of the LIN-14 temporal gradient. 
Bulged lin-4/lin-14 RNA duplexes are active in temporal gradient formation

In RNA-protein interactions such as ribosomal assembly and translational regulation, loops or bulges in RNA secondary structures have been shown to be important for protein interactions and regulatory events (Dingwall et al. 1990; Klausner et al. 1993; Hjalt and Wagner 1995). Four of the seven proposed lin-14/lin-4 RNA duplexes bulge a lin- $4 \mathrm{C}$ residue and three form thermodynamically more stable nonbulged duplexes in this region $/ \mathrm{ar}-$ row in Fig. 1B). The same set of lin-4/lin-14 RNA duplex structures in the same $5^{\prime}$ to $3^{\prime}$ order are also predicted from the C. briggsae lin-4 and lin-14 RNA sequences (Fig. 1B; Lee et al. 1993).

In addition, the most likely lin- 4 bulged $C$ nucleotide in the four putative bulged RNA duplexes is changed to a $U$ in the strong lin-4(ma161) mutant (Fig. 1B; Lee et al. 1993). The lin-4(ma161) mutation disrupts LIN-14 temporal gradient formation in vivo and by the col-10/lacZ/ lin-14(+) 3' UTR reporter gene (Fig. 3) as potently as the lin-4(e912) mutation that deletes lin-4 entirely (Lee et al. 1993). These genetic data suggest that the bulged C RNA duplexes as well as the nonbulged RNA duplexes play an essential role in LIN-14 temporal gradient formation. However, thermodynamic calculations as well as in vitro binding studies (Fig. 2; see below) suggest that if an RNA duplex per se is the essential feature for temporal gradient formation, the three more stable lin-4/lin-14 RNA duplexes would also contribute to lin-14 3' UTR mediated down-regulation.

To distinguish the role of the two types of lin-4 binding sites in temporal gradient formation, we assayed reporter genes bearing multimerized (six copies) single bulged $\mathrm{C}$ or nonbulged lin-4 binding sites (Fig. 4A). These multimerized lin-4 binding sites were assayed for temporal down-regulation in the context of the unc-54 3' UTR in both wild type and the lin-4(e912); lin-14(n179ts) background (Fig. 4B). Reporter genes with six copies of the bulged C lin-14/lin-4 RNA duplex generate a lin-4 dependent temporal gradient (120-fold temporal downregulation) that is similar to that generated by the lin14(+) 3' UTR (Fig. 4B). Reporter genes bearing multimerized lin -4 binding sites predicted to form nonbulged lin-4/lin-14 RNA duplexes show much less down-regulation that is not lin-4-dependent (Fig. 4B).

These data suggest that the bulged C lin-4/lin-14 RNA duplexes alone can confer the temporal gradient generating activity of the lin-14 $3^{\prime}$ UTR. In combination with our previous observation that a reporter gene carrying two nonbulged and one bulged C lin- 4 binding site shows intermediate temporal gradient generating activity (Wightman et al. 1993), these data suggest that multiple lin- 4 bulged $\mathrm{C}$ binding sites are essential for temporal gradient forming activity.

In vitro binding studies are consistent with the thermodynamic calculations of RNA duplex stability (Jaeger et al. 1989). RNAs bearing six copies of the bulged C or nonbulged lin-4 binding sites were incubated with ${ }^{32} \mathrm{P}$ labeled lin-4 RNA and analyzed as above (Fig. 4C). lin-
4 (+) RNA binds strongly in vitro to a lin-14 RNA bearing multimerized nonbulged lin-4 binding sites (calculated $\Delta \mathrm{G}=-23.1 \mathrm{kcal} /$ mole per RNA duplex), although it does not bind in vitro to a lin-14 RNA bearing multimerized bulged $\mathrm{C}$ lin-4 binding sites (calculated $\Delta \mathrm{G}=-17.0 \mathrm{kcal} / \mathrm{mole}$ ) (Fig. 4C). The lin-4(ma161) RNA also binds to the nonbulged lin-14 RNA, consistent with thermodynamic predictions based on the $G:: U$ base pairing (calculated $\Delta \mathrm{G}=-20.5 \mathrm{kcal} / \mathrm{mole}$ ). These results are paradoxical to the lack of temporal gradient formation by a $3^{\prime}$ UTR bearing nonbulged lin-4 binding sites and the generation of a temporal gradient by the $3^{\prime}$ UTR bearing bulged $C$ lin- 4 binding sites. The results suggest that formation of RNA duplexes is not sufficient for temporal gradient formation. Rather, the bulged $\mathrm{C}$ structural feature of the lin-4/lin-14 RNA duplex is essential for temporal gradient formation. The lack of in vitro stability of the lin-4/lin-14 RNA duplex bearing the bulged lin-4 $\mathrm{C}$ suggests that a protein factor may recognize this bulged $C$ to stabilize the lin-14/lin-4 duplex in vivo.

\section{Discussion}

The lin-4/lin-14 RNA duplex is necessary and sufficient for temporal gradient formation

The lin-14 3' UTR mediates the generation of a LIN-14 temporal gradient by post-transcriptional down-regulation of LIN-14 translation from the nontemporally regulated lin-14 mRNA (Wightman et al. 1993). The lin-4 regulatory RNA (Lee et al. 1993) also acts post-transcriptionally (Wightman et al. 1993). This lin-4-dependent post-transcriptional regulation via a lin-14 3' UTR can be conferred to an unrelated reporter gene showing that lin-4 acts via the lin-14 3' UTR (Wightman et al. 1993).

The experiments we report here show that the lin-4 RNA binds to conserved elements in the lin-14 3' UTR and that the lin-4 complementary sites of the lin-14 $3^{\prime}$ UTR are necessary for lin-14 3' UTR-mediated lin-4-dependent temporal gradient formation. In addition we show that particular lin-4/lin-14 RNA duplexes that bulge a $\mathrm{C}$ residue are sufficient to confer such temporal gradient generation activity.

\section{A bulged $C$ lin-4/lin-14 RNA duplex generates a temporal gradient}

We find that a multimerized bulged C 1 in -4 binding site is sufficient to confer the same lin-4-dependent temporal down-regulation as the lin-14 3' UTR bearing all of these multiple conserved blocks. Other experiments have shown that a $3^{\prime}$ UTR bearing three lin-4 binding sites generates only partial temporal gradient activity (Wightman et al. 1993), suggesting that more than three such sites are essential for full temporal gradient-generating activity. Because six bulged C sites are sufficient for full temporal gradient formation, it is likely that only the lin-4 complementary regions of the lin-14 $3^{\prime}$ UTR are essential for temporal gradient formation. 


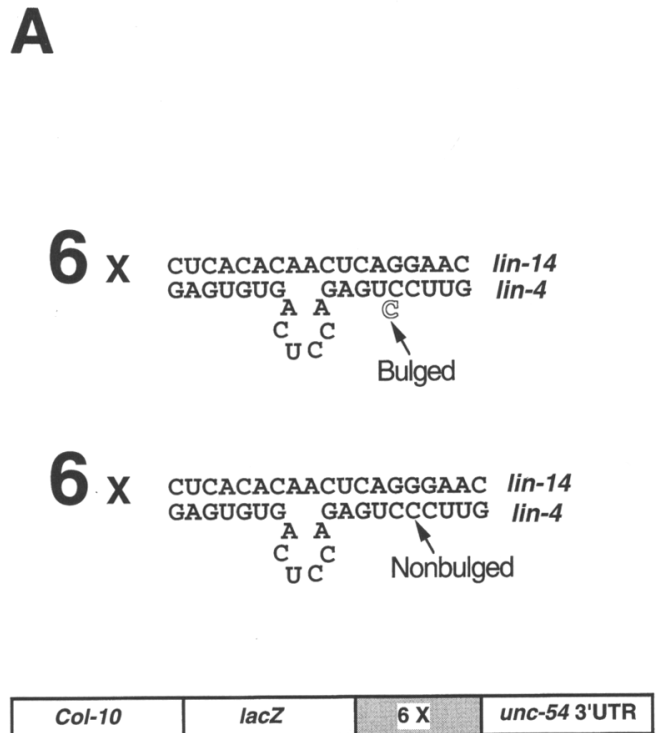

\begin{tabular}{|l|l|l|l|}
\hline Col-10 & lacZ & $6 \mathrm{X}$ & unc-54 3'UTR \\
\hline
\end{tabular}
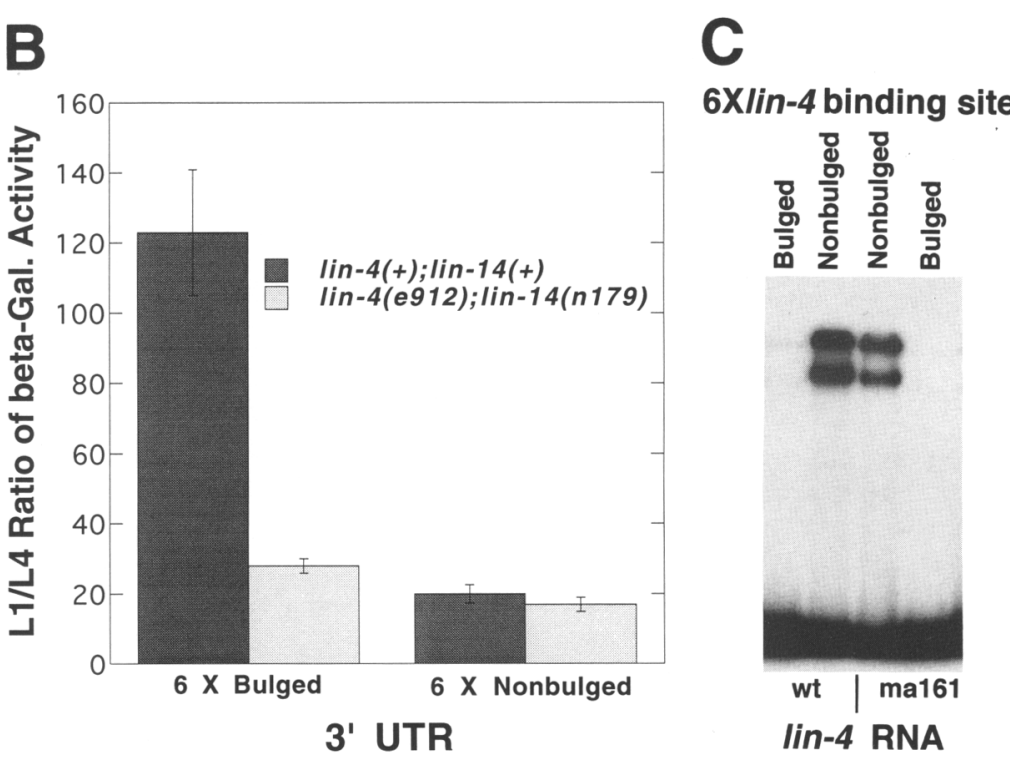

Figure 4. (A) Structure of transgenes bearing multimerized bulged or nonbulged lin-4/lin-14 RNA duplexes. Multimerized bulged C or nonbulged lin-4/lin-14 RNA duplex sites were placed downstream of lacZ in the context of unc-54 3' UTR. (B)Ratio of $\beta$-galactosidase activity of the bulged and nonbulged transgenes. lin-4-dependent temporal down-regulation of $\beta$-galactosidase activity is shown for col-10/lacZ/3' UTR reporter genes bearing six copies of the bulged C lin-4 complementary site but not in the reporter genes bearing six copies of the nonbulged site. $(C)$ In vitro binding analysis of lin-4 RNA and lin-14 RNA that has six copies of the bulged C or nonbulged lin-4 RNA binding sites. lin-14 RNAs were synthesized by T3 RNA polymerase in vitro and lin-4 RNAs were synthesized chemically. ${ }^{32}$ p-labeled lin-4 RNA was incubated with lin-14 RNA at room temperature and analyzed by a $6 \%$ native polyacrylamide gel electrophoresis. Unbound lin-4 RNA migrates to the bottom of the gel, and complexes migrate more slowly. lin-4(ma161) RNAs bind to the nonbulged lin-14 RNAs but do not bind to the bulged C lin-4 sites. The amount of RNA for each lane was normalized (data not shown).

It is surprising that only the bulged C lin-4 complementary site is sufficient for temporal gradient generation. Both the C. briggsae and C. elegans lin-14 3' UTRs bear four bulged $\mathrm{C}$ lin-4-complementary sites and three perfect duplex lin-4 complementary sites (Fig. 1B; Wightman et al. 1993), suggesting that the perfect duplex sites must also have some essential function as well. They might be necessary for down-regulation in cooperation with bulged lin- 4 binding sites, although they have no activity by themselves. For example, because the nonbulged sites are predicted to have the highest affinity, they could be the first RNA duplexes to form as lin-4 RNA levels increase (Hjalt and Wagner 1995). Such an RNA duplex may melt other lin-14 mRNA structures to expose the next most affine site, to foster cooperative binding of lin-4 RNA molecules to sites showing lower affinity, such as the bulged $\mathrm{C}$ sites. Or it is possible that more than four bulged $C$ sites may generate too steep a LIN-14 temporal gradient, which would disrupt temporal patterning.

The essential function of the bulged $\mathrm{C}$ sites is supported by the strong lin-4(ma161) mutant that is predicted to bulge a $\mathrm{U}$ rather than a $\mathrm{C}$ at these RNA duplex sites (Fig. 1B). This lin-4 mutation strongly disrupts formation of a temporal gradient by the lin-14(+) 3' UTR (Fig. 3). lin-4(ma161) is also predicted to substitute a slightly destabilizing $\mathrm{G}:: \mathrm{U}$ for a $\mathrm{G}: \mathrm{C}$ base pair in the three predicted nonbulged lin-14/lin-4 RNA duplex regions (calculated $\Delta \Delta \mathrm{G}=2.6 \mathrm{kcal} / \mathrm{mole}$ ) (Figs. 2 and
4C). The slight effect of this mutation on the perfect duplex stability, coupled with our demonstration that the bulged $\mathrm{C}$ elements are the active temporal gradient generating elements, strongly suggests that the lin-4 bulged $\mathrm{C}$ nucleotide is essential for down-regulation of lin-14 mRNA translation (Dingwall et al. 1990; Hjalt and Wagner 1995). It is possible that such bulged U sites in lin-4(ma161) cannot be recognized by protein factors that normally couple the formation of these lin-4/lin-14 RNA structures to down-regulation of lin-14 mRNA translation (Fig. 5).

In vitro binding experiments support the model that lin- 4 regulates lin- 14 by binding to the 1 in -4 complementary sites (Fig. 2). However, some of the results, although thermodynamically predicted, are paradoxical to the in vivo results. For example, the proposed bulged C RNA duplexes do not form under these in vitro conditions that allow formation of the predicted perfect duplexes (Fig. $4 \mathrm{C}$ ), but the bulged C lin-14 sites form a temporal gradient in vivo (Fig. 4B). This suggests that in vivo transiently formed bulged C RNA duplexes may be stabilized by protein factors that specifically recognize this structure. If the bulged $\mathrm{C}$ residue was essential for recognition of this RNA duplex, the bulged U lin-4(ma161)/lin-14 RNA duplexes may fail to be stabilized by the association to such a duplex binding protein. Such an unbound lin-4(ma161) RNA might be degraded; in fact the level of the lin-4(ma161) RNA is lower than in wild type ( $R$. Feinbaum and V. Ambros, pers. comm.). However, the 


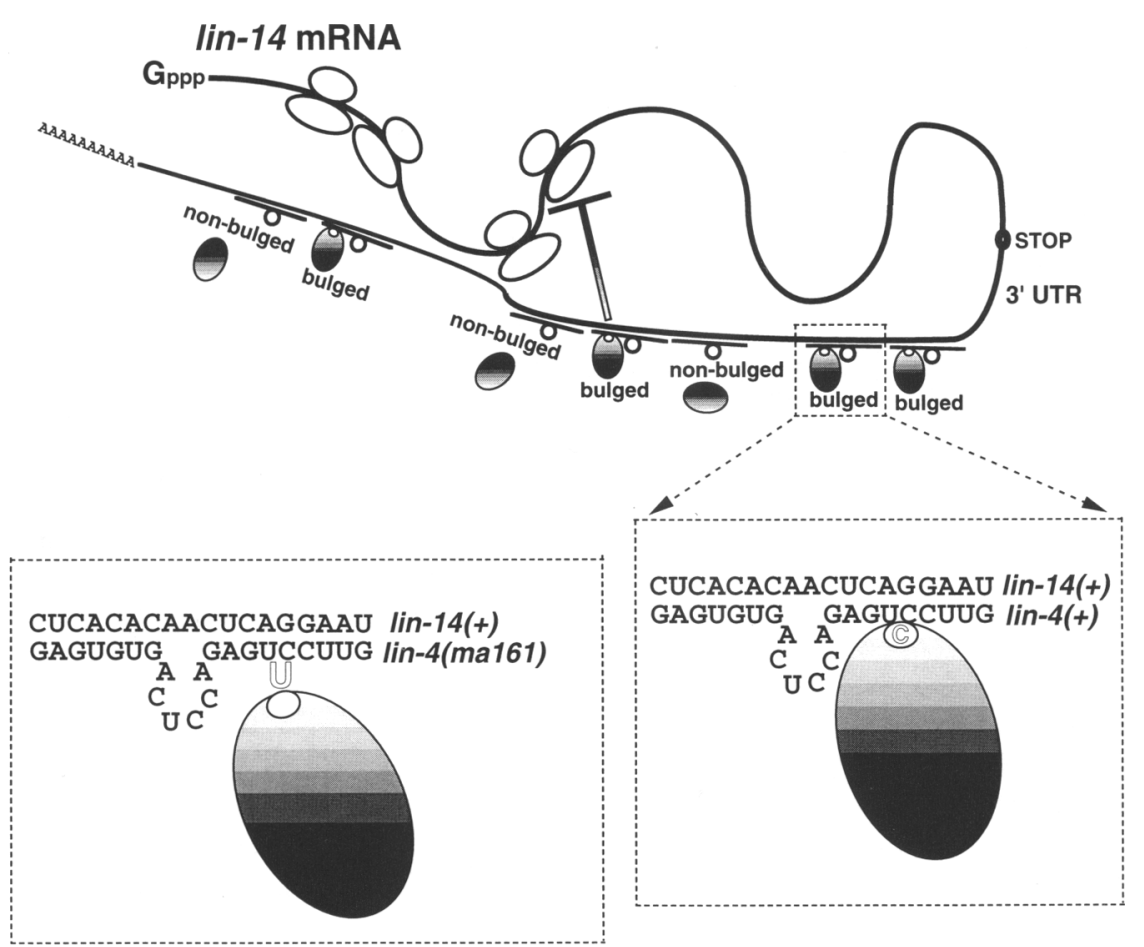

Figure 5. Model for the down-regulation of LIN-14 by formation of bulged and nonbulged lin-4/lin-14 RNA duplexes. A transacting factor recognizes the four bulged lin-4/lin-14 RNA duplexes, and these protein-RNA complexes inhibit translation of the lin-14 mRNA. However, it does not recognize the three nonbulged RNA duplexes. The factor might recognize the specific bulged $\mathrm{C}$ of the duplex (lower right) and stabilize the lin-4/lin-14 RNA duplex. However, the factor may fail to recognize a bulged $U$ in the transient lin4(ma161)/lin-14 RNA duplex (lower left). lin-4(ma161) mutation may destabilize the lin-4 RNA by a distinct mechanism, or may affect $l i n-4$ promoter activity (R. Feinbaum and V. Ambros, pers. comm.).

RNA duplexes are A-form double helices that have equally sized major and minor grooves, neither of which is wide enough to allow entry of an alpha-helical protein domain, unlike B-form DNA duplexes, which have a much larger major groove and correspondingly smaller minor groove (Weeks and Crothers 1993; Chen and Frankel 1995). Bulges and loops in RNA structures have been shown to form recognition elements for RNA-binding proteins (Predki et al. 1995; Scripture and Huber 1995). For example, the specific bulged structure in the stem region of Tar RNA is recognized by Tat protein (Puglish et al. 1992). Similarly, the lin-4 bulged C might provide a scaffold for RNA-binding proteins.

Our conclusion that all of the temporal gradient-generating activity of the lin-14 $3^{\prime}$ UTR is resident only in the lin-4 complementary regions was not predicted from the analysis of the LIN-14 gradient in 1 in- 4 null mutants and lin-14(gf) mutants. This analysis shows more LIN-14 down-regulation in the lin-4 null mutant than in the strong lin-14(n355gf) mutant (Wightman et al. 1993). This result has been confirmed qualitatively by immunofluorescence studies of LIN-14 levels (G. Ruvkun, unpubl.). One possible explanation of this difference is that lin-4 may also regulate the expression of other factors that destabilize LIN-14 (e.g., a lin-4-regulated LIN-14 cofactor or protease; Arasu et al. 1991); in a lin-4 mutant such destabilizing factors may be up-regulated, whereas in a $\operatorname{lin}-14(g f)$ mutant, they may be down-regulated by wild-type lin-4 RNA.

While the in vitro and in vivo experiments strongly suggest that the lin-4 RNA binds directly to the complementary regions of the lin-14 3' UTR, this in vivo interaction has not been demonstrated by compensatory lin-4 and lin-14 mutations (Datta and Weiner 1991; Reich et al. 1992). A compensatory lin-4 mutation (AGU to UCA; Fig. $1 \mathrm{~B}$ ) is not expected to down-regulate a wild-type lin$143^{\prime}$ UTR but should down-regulate the lin-14(7Xmut) $3^{\prime}$ UTR that bears the complementary mutations in the seven lin-4 binding sites. However, such an engineered lin-4 mutant RNA is unstable or not expressed (P. Olsen and V. Ambros, pers. comm.), precluding such a formal in vivo proof. Our proposed 1 in-4/lin-14 RNA structure is supported by the conservation between C. elegans and C. briggsae of both lin-4 and lin-14 sequences in the complementary regions. The locations and the detailed features of the proposed bulged $\mathrm{C}$ and nonbulged regions are also conserved.

Multiple blocks of conserved lin-14 3' UTR sequence between $C$. elegans and $C$. briggsae highlight elements that may be required for LIN-14 protein temporal gradient formation or other functions such as polyadenylation (Fig. 1A). The conserved blocks that are complementary to the lin-4 RNA are essential for in vitro binding of the lin-4 RNA to the lin-14 3' UTR (Fig. 2) and for in vivo temporal gradient formation (Fig. 3). The other conserved features of the lin-14 3' UTR may be binding sites for trans-acting factors that act in addition to or cooperatively with lin-4. Alternatively, they may participate in other regulatory activities of the lin-14 3' UTR /e.g., regulation in particular cell types or finer scale temporal regulation). Phylogenetically conserved elements in $3^{\prime}$ UTRs of other Drosophila and C. elegans pattern formation genes post-transcriptionally regulate gene expres- 
sion, especially during germ-line and early maternal development (Irish et al. 1989; MacDonald 1990; Ahringer and Kimble 1991; Wharton and Struhl 1991; Goodwin et al. 1993; Evans et al. 1994; Curtis et al. 1995; Murata and Wharton 1995). Proteins that bind these elements to mediate translational regulation have been identified (KimHa and Macdonald 1995; Murata and Wharton 1995; Dubnau and Struhl 1996). It is probable that the conserved elements in the lin-14 3' UTR are also recognized by regulatory proteins.

Regulatory RNAs that generate RNA duplexes have been shown to control prokaryotic plasmid replication, transposition, and gene expression (Persson et al. 1988; Wagner and Simons 1994) as well as eukaryotic viral and host gene expression (Mellits et al. 1990; Klausner et al. 1993). Given the involvement of RNA duplexes in gene regulation from bacteria to metazoans, we expect that the mechanism by which the bulged C lin-4/lin-14 RNA duplex is recognized to down-regulate translation may be general.

\section{Materials and methods}

Molecular biology

A C. briggsae genomic library in $\lambda$ Charon 4 (David Baillie, Simon Fraser University, Burnaby, British Columbia, Canada) was screened with ${ }^{32}$ p-labeled 3.8 EcoRI fragment (Wightman et al. 1991; Fig. 1) and washed at low stringency $\left(55^{\circ} \mathrm{C}, 2 \times \mathrm{SSC}\right)$ (Ausubel et al. 1993) Insert fragments were subcloned and sequenced.

For construction of a lacZ fusion gene bearing the lin-14 3' UTR with a set of three point mutations in each of the seven lin-4 complementary sites ( $7 \mathrm{Xmut}$ ), two restriction sites (StuI and HindIII) were created in the lin-14 $3^{\prime}$ UTR at positions 24567 and 25062 (Fig. 1A) by site-directed mutagenesis. Four long DNA oligonucleotide primers (nucleotides 24562-24692, 24693-24830, 24831-24956, and 24955-25066; they are numbered according to genomic sequences of cosmid $\mathrm{T} 25 \mathrm{Cl} 2$ that contains the lin-14 gene, GenBank accession no. Z66566) that substitute AGU for UCA at each of the seven lin-14/lin-4 RNA duplex regions (see Fig. 1B) were chemically synthesized and purified on a denaturing $12 \%$ polyacrylamide gel. Eight short DNA oligonucleotide primers that bridge the long DNA primers were used to join the entire set together by sequential PCR. The 3' end of the short bridging DNA primers was designed to be adjacent to a $3^{\prime}$ A to render inconsequential nonspecific addition of extra A nucleotides by Taq polymerase. The final PCR product was cloned into the StuI and HindIII sites of the lin-14 3 ' UTR and these two restriction sites were changed back to wild-type sequence by site-directed mutagenesis. The lin14(7Xmut) 3' UTR replaced the wild-type lin-14 3' UTR in the col-10/lacZ reporter gene pC10UT (Wightman et al. 1993). The sequence was confirmed.

Fusion genes with multimerized bulged and nonbulged lin- 4 binding sites were constructed by iterative cloning of DNA fragments generated by two pairs of oligonucleotide primers $\left(5^{\prime}\right.$ CTAGTACACTCACACAACTCAGGAACTTT- $3^{\prime}$ and its complementary oligomer for the bulged sites and $5^{\prime}$-CTAGTACACTCACACAACTCAGGGAACTTT- $3^{\prime}$ and its complementary oligomer for the non-bulged sites). Six copies of these lin-4 binding sites were obtained through six cycles of subcloning and subsequently placed into the col-10/lacZ/unc-54 3' UTR reporter gene.
In vitro RNA binding assay

To generate the various lin-14 RNAs for in vitro binding, subclones of wild-type lin-14 3' UTR, lin-14(7Xmut) 3' UTR, $6 \times$ bulged C lin- 4 binding site from the lin-14 $3^{\prime}$ UTR, and $6 \times$ nonbulged lin- 4 binding site from the lin-14 3' UTR were transcribed using T3 RNA polymerase and purified by precipitation, and their concentration was estimated by ethidium bromide staining after agarose gel electrophoresis. Wild-type and mutant lin-4 RNAs (5'-GUUCCCUGAGACCUCUGUGAG-3' for wild type and 5'-GUUCCUUGAGACCUCUGUGAG-3' for ma161) were chemically synthesized (Biotechnology Resource Laboratory, Yale Medical School). The in vitro transcribed lin14 RNAs $(0.2 \mu \mathrm{g})$ were incubated with ${ }^{32} \mathrm{P}$-labeled lin-4 RNA at room temperature for $2 \mathrm{hr}$ in $20 \mathrm{mM}$ HEPES at pH 8.0, $5 \mathrm{~mm}$ $\mathrm{MgCl}_{2}, 5 \%$ glycerol, $10 \mathrm{mg} / \mathrm{ml}$ tRNA of Escherichia coli, 1 unit RNAse inhibitor. This mixture was resolved on a native $1 \%$ agarose or $6 \%$ polyacrylamide gel $\left(\frac{1}{2} \times\right.$ TBE buffer $)$ at $100 \mathrm{~V}$ for $1.5 \mathrm{hr}$. The gel was dried and exposed to film.

\section{Temporal gradient assays}

col-10/lacZ/lin-14 3' UTR fusion genes were coinjected with the marker plasmid pRF4 as described previously (Mello et al. 1991, Wightman et al. 1993). Between 1 and 3 independent lines were obtained for each construct. The same fusion gene arrays assayed for $\beta$-galactosidase activity in wild type were crossed into lin-14(n179ts) or lin-4(e912);lin-14(n179ts) genetic backgrounds (Ambros 1989) for those assays. Thus the same arrays were tested in $\operatorname{lin}-4(e 912)$ and wild type.

Staged preparations from transformed strains bearing the various lac $Z$ constructs were obtained by treating each strain with alkaline hypochlorite to isolate a population of eggs (Wood 1988). Eggs were allowed to hatch overnight in M9 buffer to yield a pure population of L1 stage animals. Some of this preparation was frozen and the rest was fed $E$. coli strain XLl-blue (which does not produce $\beta$-galactosidase activity) until the L4 stage. Bacteria in the gut were removed by multiple rinses with sterile distilled water.

$\beta$-galactosidase activity was assayed using a chlorophenol red- $\beta$-D-galactopyranoside substrate (CPRG, Boehringer Mannheim) (Simon and Lis 1987). Approximately $20 \mu$ l of staged animals were sonicated for $10 \mathrm{sec}$ using an Ultrasonics W-375 Sonicator in $500 \mu$ l of assay buffer $(50 \mathrm{mM}$ potassium phosphate at $\mathrm{pH} 7.5,1 \mathrm{~mm} \mathrm{MgCl} 2,1 \mathrm{~mm}$ Pefabloc SC, Boehringer Manheim) and spun for $5 \mathrm{~min}$ in a microfuge at $4^{\circ} \mathrm{C}$. Supernatant was transferred to a disposable cuvette, additional assay buffer added to a total of $200 \mu \mathrm{l}$, and $300 \mu \mathrm{l}$ of $1 \mathrm{mg} / \mathrm{ml} \mathrm{CPRG} \mathrm{in} \mathrm{assay} \mathrm{buffer}$ added to each. Samples were mixed and incubated at room temperature. $\mathrm{A}_{574}$ was measured on a Beckman spectrophotometer every $30 \mathrm{~min}$ for $2 \mathrm{hr}$. Activity was calculated by dividing the change in $\mathrm{A}_{574}$ over time by the amount of total protein of transgenic animals in each extract (determined by Bradford Method, Bio-Rad). Multiple dilutions of test extracts showed that this assay yielded results that increased linearly with the amount of extract over the range of O.D. used in these experiments. Extracts made from E. coli strain XL1-Blue, on which worms were fed, or wild-type C. elegans fed this strain yielded no detectable activity in this assay.

\section{Acknowledgments}

We thank the Ambros group for helpful discussions. We thank lab members for helpful comments, especially those from Rhonda Feinbaum, Brenda Reinhart, and Frank Slack. We thank Joe Gatto for assistance in sequencing. I.H. is supported by post- 
doctoral fellowship from Cancer Research Fund of the Damon Runyon-Walter Winchell Foundation (DRG-1287). This work was also supported by American Cancer Society Faculty Research Award (FRA-382) to G.B.R., and National Institutes of Health grant GM44619.

The publication costs of this article were defrayed in part by payment of page charges. This article must therefore be hereby marked "advertisement" in accordance with 18 USC section 1734 solely to indicate this fact.

\section{References}

Ahringer, J. and J. Kimble 1991. Control of the sperm-oocyte switch in Caenorhabditis elegans hermaphrodites by the fem-3 3' untranslated region. Nature 349: 346-348.

Ambros, V. 1989. A hierarchy of regulatory genes controls a larva-to-adult developmental switch in C. elegans. Cell 57: 49-59.

Ambros, V. and H. R. Horvitz. 1984. Heterochronic mutants of the nematode Caenorhabditis elegans. Science 226: 409416.

Arasu, P., B. Wightman, and G. Ruvkun. 1991. Temporal regulation of $l i n-14$ by the antagonistic action of two other heterochronic genes, lin-4 and lin-28. Genes \& Dev. 5: 1825 1833.

Ausubel, F.M., R. Brent, R.E. Kingston, D.D. Moore, J.A. Smith, J.G. Seidman, and K. Struhl. 1993. Current protocols in molecular biology. Wiley Interscience/Greene, New York, N.Y.

Chalfie, M., H.R. Horvitz, and J.E. Sulston. 1981. Mutations that lead to reiterations in the cell lineages of C. elegans. Cell 24: 59-69.

Chen, L. and A.D. Frankel. 1995. A peptide interation in the major groove of RNA resembles protein interactions in the minor groove of DNA. Proc. Natl. Acad. Sci. 92: 5077-5081.

Curtis, D., J. Apfeld, and R. Lehmann. 1995. nanos is an evolutionarily conserved organizer of anterior-posterior polarity. Development 121: 1899-19140.

Datta, B. and A.M. Weiner. 1991. Genetic evidence for base pairing between U2 and U6 snRNA in mammalian nRNA splicing. Nature 352: 821-824.

Dingwall, C., I. Ernberg, M.J. Gait, S.M. Green, S. Heaphy, J. Karn, A.D. Lowe, M. Singh, and M.A. Skinner. 1990. HIV-1 tat protein stimulates transcription by binding to a U-rich bulge in the stem of the TAR RNA structure. EMBO $I$. 9: 4145-4153.

Dubnau, J. and G. Struhl. 1996. RNA recognition and translational regulation by a homeodomain protein. Nature 379: 694-699.

Evans, T.C., S.L. Crittenden, V. Kodoyianni, and J. Kimble. 1994. Translational control of maternal glp-1 mRNA establishes an asymmetry in the C. elegans embryo. Cell 77: 183194.

Goodwin, E.B., P. Okkema, T. Evans, and J. Kimble. 1993. Translational regulation of tra-2 by its $3^{\prime}$ untranslated region controls sexual identity in C. elegans. Cell 75: 329-339.

Heschl, M.F. and D.L. Baillie 1990 Functional elements and domains inferred from sequence comparisons of a heat shock gene in two nematodes. J. Mol. Evol. 31: 3-9.

Hjalt, T.A. and E.G. Wagner. 1995. Bulged-out nucleotides in an antisense RNA are required for rapid target RNA binding in vitro and inhibition in vivo. Nucleic Acids Res. 23: 580-587.

Irish, V., R. Lehmann, and M. Akam. 1989. The Drosophila posterior group gene nanos functions by repressing hunchback activity. Nature 338: 646-648.

Jaeger, J.A., D.H. Turner, and M. Zuker. 1989. Improved predic- tions of secondary structures for RNA. Proc. Natl. Acad. Sci. 86: 7706-7710.

Kim-Ha, J. and P.M. Macdonald. 1995. Translational regulation of oskar mRNA by bruno, an ovarian RNA-binding protein, is essential. Cell 81: 403-412.

Klausner, R.D., T.A. Rouault, and J.B. Harford. 1993. Regulating the fate of mRNA: The control of cellular iron metabolism. Cell 72: 19-28.

Lee, C., R. Feinbaum, and V. Ambros. 1993. The product of the heterochronic gene lin- 4 is a small RNA with antisense complementarity to lin-14. Cell 75: 843-854.

MacDonald, P.M. 1990. bicoid mRNA localization signal: Phylogenetic conservation of function and RNA secondary structure. Development 110: 161-171.

Mellits, K.H., M. Kostura and M.B. Mathews 1990. Interaction of adenovirus VA RNAl with the protein kinase DAI: Nonequivalence of binding and function. Cell 61: 843-852.

Mello, C., J.M. Kramer, D. Stinchcomb, and V. Ambros. 1991. Efficient gene transfer in C. elegans: Extrachromosomal maintenance and integration of transforming sequences. EMBO I. 10: 3959-3970.

Murata, Y. and R.P. Wharton. 1995. Binding of pumilio to maternal hunchback mRNA is required for posterior patterning in Drosophila embryos. Cell 80: 747-756.

Nüsslein-Volhard, C. 1991. Determination of the embryonic axes of Drosophila. Development (Suppl.) 1: 1-10.

Persson, C., E.G. Wagner, and K. Nordstrom. 1988. Control of replication of plasmid R1: Kinetics of in vitro interaction between the antisense RNA, CopA, and its target, CopT. EMBO I. 7: 3279-3288.

Predki, P.F., L.M. Nayak, M.B. Gottlieb, and L. Regan. 1995. Dissecting RNA-protein interactions: RNA-RNA recognition by Rop. Cell 80: 41-50.

Puglish, J.D., R. Tan, B.J. Calnan, A.D. Frankel, and J.R. Williams. 1992. Conformation of the TAR RNA-arginine complex by NMR spectroscopy. Science 257: 76-80.

Reich, C.I., R.W. VanHoy, G.L. Porter, and J.A. Wise. 1992. Mutations at the $3^{\prime}$ splice site can be suppressed by compensatory base changes in U1 snRNA in fission yeast. Cell 69: $1159-1169$.

Rushlow, C., K. Han, J. Manley, and M. Levine. 1988. The graded distribution of dorsal morphogen is initiated by selective nuclear transport in Drosophila. Cell 59: 1165-1177.

Ruvkun, G. and J. Giusto. 1989. The Caenorhabditis elegans heterochronic gene lin-14 encodes a nuclear protein that forms a temporal developmental switch. Nature 338: 313319.

Scripture, J.B. and P.W. Huber. 1995. Analysis of the binding of Xenopus ribosomal protein $\mathrm{L} 5$ to oocyte $5 \mathrm{~S}$ rRNA. The major determinants of recognition are located in helix III-loop C. J. Biol. Chem. 270: 27358-27365.

Serano, T.L. and R.S. Cohen. 1995. A small predicted stem-loop structure mediateds oocyte localization of Drosophila K10 mRNA. Development 121: 3809-3818.

Simon, J.A. and J.T. Lis. 1987. A germline transformation analysis reveals flexibility in the organization of heat shock consensus elements. Nucleic Acids Res. 15: 2971-2988.

Wagner, E.B.H. and R.W. Simons. 1994. Antisense RNA control in bacteria, phages, and plasmids. Annu. Rev. Microbiol. 48: 713-742.

Wang, C. and R. Lehmann. 1991. Nanos is the localized posterior determinant in Drosophila. Cell 66: 637-647.

Weeks, K.M. and D.M. Crothers. 1993. Major groove accessibility of RNA. Science 261: 1574-1577.

Wharton, R. and G. Struhl. 1991. RNA regulatory elements mediate control of Drosophila body pattern by the posterior 
Ha et al.

morphogen nanos. Cell 67: 955-967.

Wightman, B., T.R. Bürglin, J. Gatto, P. Arasu, and G. Ruvkun. 1991. Negative regulatory sequences in the lin-14 3'-untranslated region are necessary to generate a temporal switch during Caenorhabditis elegans development. Genes \& Dev. 5: 1813-1824.

Wightman, B., I. Ha, and G. Ruvkun. 1993. Post-transcriptional regulation of the heterochronic gene lin-14 by lin-4 mediates temporal pattern formation in C. elegans. Cell 75: 855-862.

Wood, W.B. 1988. The nematode Caenorhabditis elegans. Cold Spring Harbor Laboratory, Cold Spring Harbor, NY.

Xue, D., M. Finney, G. Ruvkun, and M. Chalfie. 1992. Regulation of the mec-3 gene by the $C$. elegans homeoproteins UNC-86 and MEC-3. EMBO I. 11: 4969-4979.

Zucker-Aprison, E. and T. Blumenthal. 1989. Potential regulatory elements of nematode vitellogenin genes revealed by interspecies sequence comparison. J. Mol. Evol. 28: 487-496. 


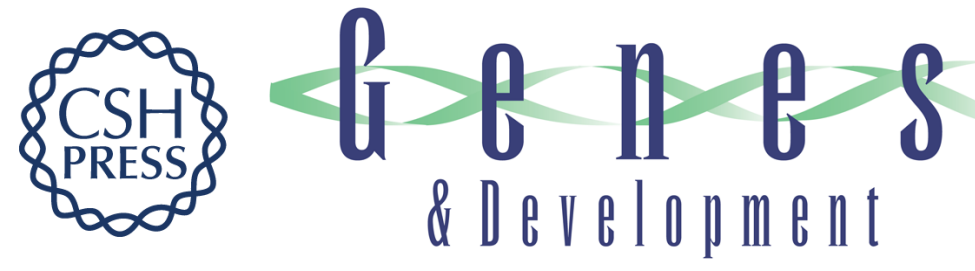

\section{A bulged lin-4/lin-14 RNA duplex is sufficient for Caenorhabditis elegans lin-14 temporal gradient formation.}

I Ha, B Wightman and G Ruvkun

Genes Dev. 1996, 10:

Access the most recent version at doi:10.1101/gad.10.23.3041

References This article cites 41 articles, 11 of which can be accessed free at:

http://genesdev.cshlp.org/content/10/23/3041.full.html\#ref-list-1

License

Email Alerting

Service

Receive free email alerts when new articles cite this article - sign up in the box at the top right corner of the article or click here.

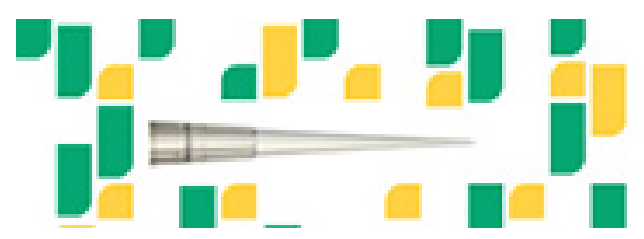

Focused on your science.

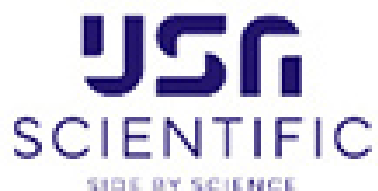

Copyright (c) Cold Spring Harbor Laboratory Press 